\title{
Pre and Post COVID-19 Sentiment Analysis of Consumers for OTT Platforms
}

\author{
Raka Ghosh Dastidar ${ }^{1}$ \\ ${ }^{1}$ Symbiosis Institute of Operations Management, Nasik, Symbiosis International (Deemed University), Pune, India
}

\begin{abstract}
The entire media and entertainment industry are going through unprecedented changes in this age of digital disruption. The rise of the Over-The-Top (OTT) platform has transformed consumer experience and expectations. OTT players (Netflix, Amazon Prime Video, Hulu, Disney+, etc.) offer a wide array of diverse choices of content while enabling consumers to choose their content, watching time, and place independently. OTT platforms and digital media have already seen increasing popularity across locations and demographics. Adding to the momentum, due to the COVID-19 pandemic, social lives have shifted to online, people are restricted to their homes, so inclination towards OTT and digital media has accelerated significantly. Consumer behavior is undergoing profound change as the world adjusts to this new normal. This study aims to understand the changing consumer behavior towards the adaptation of the OTT platform during the COVID-19 pandemic.
\end{abstract}

\section{KEYWORDS:}

Over-The-Top, digital media, Netflix, entertainment industry, COVID-19, OTT players

Article Received: 10 August 2020, Revised: 25 October 2020, Accepted: 18 November 2020

\section{Introduction}

In recent times COVID-19 outbreak has resulted in significant economic, mental, and physical health consequences. Due to social distancing measures employed by several countries around the globe, the entire dynamic of how people communicate and work, how people live and interact with each other, how people move around, and travel has been changed. While adherence to these lockdown measures psychological traits like boredom, anxiety, stress, insecurities took a front seat for many of us.

The COVID-19 pandemic is disrupting the way business functions. When comes to Entertainment and Media business, coronavirus creates both challenges and opportunities. Due to social distancing norms, home media consumption increased and big broadcasting events like live sports, theatre movie release have been postponed or cancelled. In response to all the difficulties, digital media has come up with some new normal aspects of the entertainment business.

Global Media and entertainment have seen swift changes over the year. Digital disruptions have changed the way we experience content. With higher internet penetration and easy access to fresh content, the OTT industry has seen a drastic increase in revenue. OTT stands for "over- thetop," which essentially means that audio-visual entertainment contents are delivered over the top of traditional types of TV receivers such as satellite, set-top boxes, and dishes to viewers by way of an on-demand delivery system (Urgelles, 2017).

Some of the major Global players of OTT video platforms are HBO, YouTube Tv, Amazon, Hulu, Netflix, Apple, Crackle, Facebook watch, Sling, Disney, etc. India's first OTT video platform was BIGFlix by Reliance entertainment which was launched in 2008. Then Digivive launched the first OTT Video platform which could be accessed through a mobile phone called nexGTv which served both live TV and video on demand, in 2010. NexGTv was the first OTT video platform to live stream IPL in the years 2013 and 2014. In India, around 40 providers of over-the-top media services (OTT) are present in the market.

PwC, Global Entertainment, and Media Outlook report 2019 suggested that the OTT Video market will grow almost double between 2019 to 2029 and will represent 72.8 billion industry by 2023 and will grow with almost $14 \%$ CAGR

\subsection{Different types of content go well with OTT platform}

Though general perception about the OTT platform largely moves around VOD(Video-ondemand) services and the concept of the OTT technology \& innovation covers a much wider range of content, as mentioned below. 
- Video: Online video streaming is the most popular type of content, OTT platform provides. There are many platforms like Netflix, Hotstar offer a large variety of video content based on subscription-based or free membership business model.

- Audio: Along with online video streaming audio streaming is another important content OTT platform delivers. Podcast, the internet radio station is a well-known example of it.

- VOIP: Voice calling service is also another significant value addition by the OTT platforms, which is driven by the internet facility.

- Messaging: Nowadays different instant internet-based messaging platform (i.e. Skype, WhatsApp) is there which successfully reduce the use of the mobile SMS feature(Gaoshinde, 2020).

\subsection{Different business models for OTT Services}

In this competitive age like any other industry OTT also revolved around different innovative business models that solely depend on the customer segment, market condition, and present competition. The following are the options present in the current market.

- Subscription-based: Users need to pay a periodical (weekly/monthly or annual) fee to avail of the service.

- Usage/Transaction: Users need to pay for a particular resource or service they are using.

- Advertisements: Supplier sells their ads over the OTT platform while users are viewing any content over the OTT platform.

- Freemium: This is a hybrid model where some basic content or features are free for any user, but some content or access is offered at a price.

- Donations: There are some OTT platforms (Wikipedia), which are funded by donations.

- Monetization of Information: Users' data is disclosed, and suppliers monetize user information(Baldry et al., 2014).

Over the years significant changes had happened in different OTT platforms. Traditional telephone service is now facing a tough competition with OTT communication and SMS service replaced by different OTT players like Hike, WhatsApp, etc. Thus, traditional Telcom service provider is challenged in some of their business lines like long-distance calls, and SMS service. Also, the traditional media is facing huge competition by new OTT services providers for theircontemporary approach of audio and video streaming services. Today's consumers prefer "on- demand" experience. So, we can say there are a consumer preference shift from traditional entertainment \& media to "on-demand" media. The rise of OTT platforms and the ease of access to mobiles have drastically transformed consumer preferences towards general entertainment provided by the traditional TV industry. According to a study by TechARCUnomer, 4 out of 5 smartphone users have at least one OTT entertainment platform which is $80 \%$ of whole smartphone subscribers (Khatri, 2019). In 2018 the OTT platform revenue in India was evaluated to be 638 million this was majorly due to driving digital media consumption on mobile devices and the evolution of smart TV which allows OTT platforms to broadcast on television via the use of WiFi. Both global and Indian OTT players are providing multiple choices around content consumption to the consumer, due to which consumer preference is shifting. Domestic OTT players like Hotstar have made disruptions to how consumers used to watch the sports content. According to figures released by ICC in 2019, the world cup semi-final between India and New Zealand made a record with

25.3 million people live streaming the match on Hotstar (Rashid, 2019).

COVID-19 pandemic has restricted live entertainments and film business due to several social distancing laws and lockdown policies imposed by the government. Like many other countries In India government extended lockdown for more than 4 months resulted in a huge financial crisis in the film industries due to the inability to release movies within cinema halls. So, by following the classic rule "necessity is the mother of invention" this pandemic situation creates a brand-new way of a cinema release in OTT platforms. This move of many filmmakers resulting in prominent engagement of OTT media platforms in Movie release even after the removal of lockdown Policies.

According to a report published by Reuters, Netflix's global total has reached 182.9 million from January to March (Richwine \& Rana, 2020).It shows that people are bingeing on shows 
like Money Heist, Dark, Love, and Blind, etc. This pandemic had contributed significantly to the already changing behavioral patterns of consumer abut digital consumption.

\section{Objective}

The primary objective of this paper is to understand consumer perception about the OTT platform during the pre-COVID-19 phase and post COVID-19 phase and analyze current consumer engagement with popular OTT players, through sentiment analysis.

\section{Literature Review}

Over-The-Top service providers deliver video, audio, and other type of content over the internet and eliminate the traditional telecom operator's network. As OTT players do not need specific technology and business affiliations with telecom network operators, they are familiar with the term "Over-The-Top" (OTT) applications(Kokaram et al., 2015).

According to Herrero, the distribution of audiovisual content over the Internet is a great way to realize economies of scale and effective broadcasting strategies. But the most difficult part for the telecom players is the fact that these OTT players offer services that are close substitutes to their offerings and are posing a significant threat to their revenues. The wide range of variety of services and applications offered by OTT players is tempting the customers away from the telecom players (Herrero-Subías, 2003).

(Budzinski \& Lindstädt-Dreusicke, 2018) mentioned three characteristics of the OTT platform in contrast with the traditional entertainment medium. Firstly, OTT significantly reduces the

distribution costs and offer an open platform in which creators can share their content through an online medium (e.g. YouTube, Hotstar) and reach to a large number of audiences across demographics easily. Secondly, platforms like OTT successfully bridges the gap between the customer and content provider through real-time feedback (e.g. reviews, comments, likes, ratings). In this analytical age, feedback data plays an important role in an overall understanding of customer demand and delivering expected outcomes well. Lastly, OTT enhances the customer experience by making content available on various devices like mobile laptops, tablets, etc. Witnessing the outcome of a thirty-country study, (Noam, 2016) concluded that concentration levels for mobile wireless and other "network media" are "astonishingly high". While the data for content media is mixed, the trend of adopting OTT is increasing.

A study conducted in India reveals that OTT platform is becoming popular day by day and youth prefer to watch the content of their own choice on OTT platform, because of its multifaced feature and flexibility and wide range of variety (P. Singh, 2019). So, entertainment trends are changing due to Online platforms. People are shifting from traditional channels to the OTT platform because of the more personalized experience and that increases the penetration of mobile computing devices. The mobile phone provides more convenience of watching preferred content at anytime and anywhere rather than smart TV, laptops, and other devices. We can say that OTT (over-the-top) platforms have challenged the Indian entertainment media. Subscription-based OTT platforms like Amazon Prime, Hotstar, NextG have become popular over the past years and replacing traditional Television media for progressive Indians (Kohli, 2019).

Worldwide digital media is projected to increase to $\$ 118$ bn by 2021 with a compound annual growth rate of $11 \%$ between 2016 and 2021 (Lacy, 2017).

A recent survey by InMobi states that in this COVID-19 situation $46 \%$ of consumers are preferring to watch content online. According to the study, OTT services could evolve drastically in upcoming days as the traditional television channels lack in terms of the latest content. In such an unprecedented time, people are stressed and willing to watch fresh content. To leverage the benefit from this increasing pattern of demand OTT platforms such as Zee5 and VOOT have offered few contents free. Due to this, Zee5 has achieved an $80 \%$ increase in subscription and over $50 \%$ time spend recently. Urban drama, thrillers, and young romance are the top-performing genres on the platform. The number of viewership for the movies has seen a significant increase over the last couple of months during the ongoing pandemic situation, across many OTT platforms. Movie streaming services MIBI has witnessed a $28 \%$ rise in viewership in March, compared to 
February. In the ongoing COVID-19 pandemic situation consumption of OTT platforms has increased and surprisingly despite the ongoing economic crisis subscription trend has not been affected (D. Singh, 2020).

The first stage of the internet revolution was introducing a wireline environment. This facility of the web became available on laptops, PCs, and other electronic devices. Offering such as eCommerce, OTT Voice service came into the picture of modern life. The next stage of the internet revolution is wireless and is being driven by the domination of smartphones. Nowadays market boundaries are becoming a blur and new players are entering with innovative business models. Internet and OTT services allow these players to achieve benefits from economies of scale far beyond those of traditional telecom operators. This media market dynamism has the regulatory norms struggling to sustain in the competition space. One after another issue regarding regulation keeps coming up and the market is influenced by just in time principals (Baldry et al., 2014).

This changing market dynamic indicated that Just like a coin has two sides, the OTT platform also has a flip side of it. Along with a high pace of market penetration OTT has to bring differences amongst family members as well as individuals or we can say generations. In the previous era family use to go out have fun and watch a long-awaited movie on the television or in a theatre. But nowadays, the same family would prefer ordering food online through some web application and watch some specific video content over internet applications. This phenomenon is mostly seen in the young population of the country, which has led to creating gaps amongst family members as many of the senior citizens often complain that their children or grandchildren do not spend enough time with them(Baldry et al., 2014).

OTT Media has also underlined several data consumption issues. Most data plans have a limit on data usage, after the predefined limit volume tariff or limited data transmission speed applies. Due to the latest technology High-definition Baldry, Steingröver, \& Hessler, 2014 capable devices come on the market and data limits are quickly reached. This resulted in "bill-shock", where users need to pay fat bills as a result of streaming data. The perfect resolution of the issue is currently an open debate(Baldry et al., 2014).

The above data points prove that despite the wide variety of content and becoming a close substitute to the traditional entertainment medium there are certain challenges attached to the OTT platform.

Based on an analysis of how Netflix impacted television industry in Hong Kong shows that customer viewing habit is changing, and quality of content plays an important role while selecting the mode of entertainment beside this the study also suggest that people are interested in OTT video platform, but they hesitate to pay subscription fees (Madhani \& Nakhate, 2020). So, there is a need to understand what exactly consumer is thinking about OTT platform and are they ready to adopt this latest entertainment media trend or not.

Previously consumers use to rely on the other's opinions like specialized magazines and friends' recommendations and while making a buying decision. But along with the growth of digital technology and internet facilities, information sharing pattern has been shifted tremendously (Cambria et al., 2013)

Nowadays consumer follows online rating app, blogs, social networks, forums, and contentsharing sites, etc to understand the effectiveness of a product or service. Many reports and researches suggested that nowadays using various social media platform is considered as the best way to grow a business(Edosomwan et al., 2011). These social platforms contain a huge amount of opinion about almost each and every possible product or service. But processing these data and drawing meaningful business insights is difficult, so here also technology has brought some advanced intelligent data analysis methods like sentiment analysis, natural language processing (NLP), etc, through which we can understand consumer expectation in a structured manner( $\mathrm{Li} \&$ $\mathrm{Wu}, 2010)$.

The literature reviews, provide insights about how the OTT platform is transforming the entertainment industry but still, there are some areas where some gaps exist in researches like understanding consumer mindset towards the adaption of On-demand-entertainment media in the pre-COVID-19 era and COVID-19 era. 


\section{Methodology}

In this digital age usage of social media is growing rapidly and a good number of people share their opinions and experiences through these sites. By using one of the great engagement social platforms, Twitter, data was collected through web scraping and done sentiment analysis about the consumer mindset towards the OTT platform during this COVID-19 situation.

Twitter is an American social networking and blogging service where users can post their opinion or tweets or communicate with other users, comment on other's tweets, use popular hashtags to reach out to a wider audience. Twitter is an informative social platform and has a constructive culture of discussion compared to other social media platforms. That is why twitter data was chosen for further analysis.

Sentiment analysis is a method of data analysis that provides valuable insights about what people are thinking about a particular topic. Through sentiment analysis polarity classification and emotion classification of the people were done to understand what people are thinking about OTT platform adoption.

\section{Tools Used}

Different programming languages (i.e. Python, R) platform (Anaconda- Jupitar notebook) and data analysis methods (sentiment analysis, polarity check, emotion classification) are used for the research purpose.

Web scraping is used to fetch multiple tweets related to the OTT media platform. Web Scrapping is an automatic data collection method from the online platform. By using tweepy, python library, the twitter account was linked with the coding platform (Jupyter) and data is fetched in a data frame. Finally, fetched data saved in a csv format for further analysis.

For sentiment analysis and data visualization $\mathrm{R}$ programming language is used. $\mathrm{R}$ programming is a very user-friendly programming language to perform any statistical analysis. In the R-studio packages like syuzhet, lubridate, ggplot2, scales, reshape2, dplyr have to be added for performing the statistical analysis.

\section{Data collection}

This research paper is mainly based on secondary research, where the data has been collected from Twitter using a web scraping technique to understand the sentiment of people in the preCOVID era and during the COVID-19 era regarding OTT platforms. For the research purpose, created a developer account in app.twitter.com to link the python platform (Jupyter Notebook) with the twitter account. At first, comparative sentiment analysis was done among pre- COVID 19 phase, Lockdown phase, and post Lockdown phase considering individual phase-wise dataset of 300 sample data each. For further analysis of the people's perception of OTT platforms through word cloud generation technique, 650 tweets were collected using relevant keywords (i.e. OTT, Netflix, Amazon prime video, VOD, SVOD, Voot, YouTube, Disney+, Hotstar) and hashtags(i.e. \#ott, \#bingewatch, \#webseries) related to Over-TheTop media service. Identified the top 3 OTT players, by using the word cloud and collected three individual datasets for each of these players containing 200 sample data each.

\section{Data Pre-Processing}

Data Pre-processing or data cleaning is a necessary step in the analysis. That is why in the pre- processing only the body of the text of the tweets has been extracted from the entire file using the R-programming language. After that, in the data cleaning stage punctuation, numbers, URLs, stopwords, white space were removed since these are not required for the analysis.A small sample of data before data cleaning and after data cleaning is shown below. 


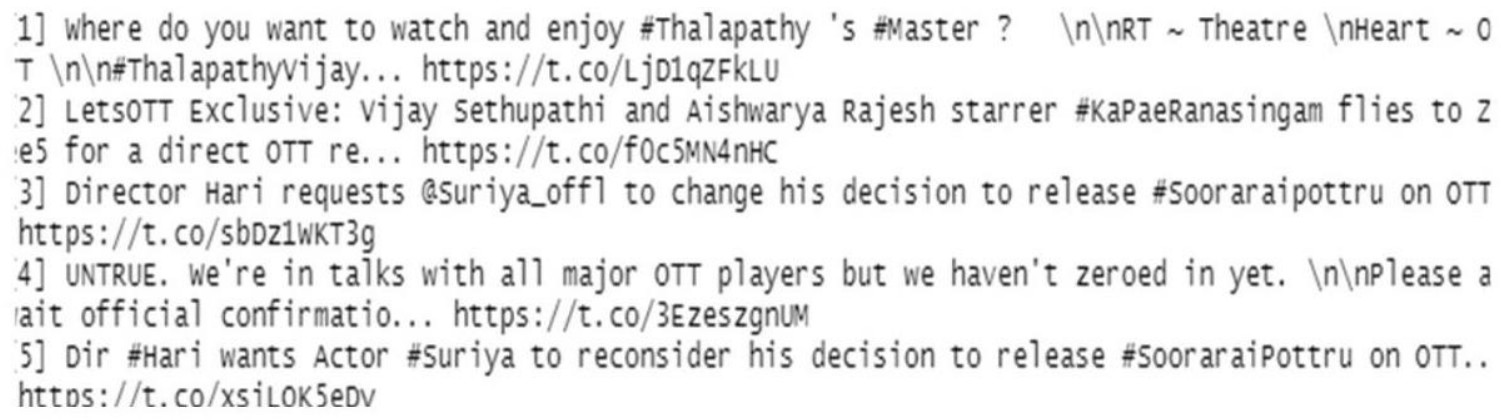

Figure 1: Before Data Cleaning of the Twitter Data

\author{
[1] want watch enjoy thalapathy s master rt theatre heart ott thalapathyvijay \\ [2] letsott exclusive vijay sethupathi aishwarya rajesh starrer kapaeranasingam flies zee direc1 \\ ott re \\ [3] director hari requests suriyaoffl change decision release sooraraipottru ott \\ [4] untrue talks major ott players havent zeroed yet please await official confirmatio \\ [5] dir hari wants actor suriya reconsider decision release sooraraipottru ott
}

\title{
Figure 2: After Data Cleaning of the Twitter Data
}

Sentiment Analysis

Consumer behaviour is important part for any business model and to understand the consumer behaviour and customer mindset sentiment analysis plays a very important role. Using sentiment analysis, understanding of what the customer thinking, whether they have positive or negative sentiment towards a particular product or service can be gained. In this paper word cloud, polarity classification and emotion classification are used for in dept sentiment analysis to draw insights about the consumer perception of OTT media platform and OTT players.

\section{Measurement}

The data generated from the data cleaning stage is the actual input for the sentiment analysis and the data were analyzed further through $\mathrm{R}$ programming.

\subsection{Word Cloud Generation}

Word cloud is a cluster of words where the most frequent word has a bigger size and with a decrease in frequency size of the words are also decreased. In the first step, the most frequently used terms in the data set were identified by text mining, while searching for the OTT platform using the term-document matrix. It helps us to find out keywords and important thoughts that come to the consumer mind while they heard about the OTT platform.

\subsection{Polarity Checking}

It is the measurement of whether the speaker expresses a positive or negative opinion. It is useful to check whether a statement is positive, negative, or neutral. The percentage of people inclined to a certain Polarity is plotted in a bargraph. Polarity value varies from -1 to +1 range. Where ' +1 ' represents strong positivity,' $\mathrm{O}$ 'represents neutral, and '-1 'represents strong negativity. For the research purpose, the dataset(tweets) is categorized into 3 segments following the below described scale.

\begin{tabular}{|l|l|}
\hline Polarity score & Statement Sentiment \\
\hline$>0$ & Positive \\
\hline 0 & Neutral \\
\hline$<0$ & Negative \\
\hline
\end{tabular}

\subsection{Emotion Categorization}

Emotion categorized of the tweets is done and classified into a 10-point scale mentioned below 
and plotted into a bar-graph, which is useful in visual analysis.
(1) Positive
(6) Joy
(2) Negative
(7) Fear
(3) Trust
(8) Disgust
(4) Surprise
(9) Anticipation
(5) Sadness
(10) Anger

\section{Finding and Result}

In this COVID-19 reality, as the world is adjusting adoption of OTT media are increasing day by day. In this paper, consumer sentiment is analyzed in three different timeframes for understanding changing consumer behaviours during COVID-19 toward OTT platforms.

Below are the three phases considered for the sentiment analysis

1. Pre CODIV-19 Phase

2. Lockdown Phase due to COVID-19

3. Post Lock Phase

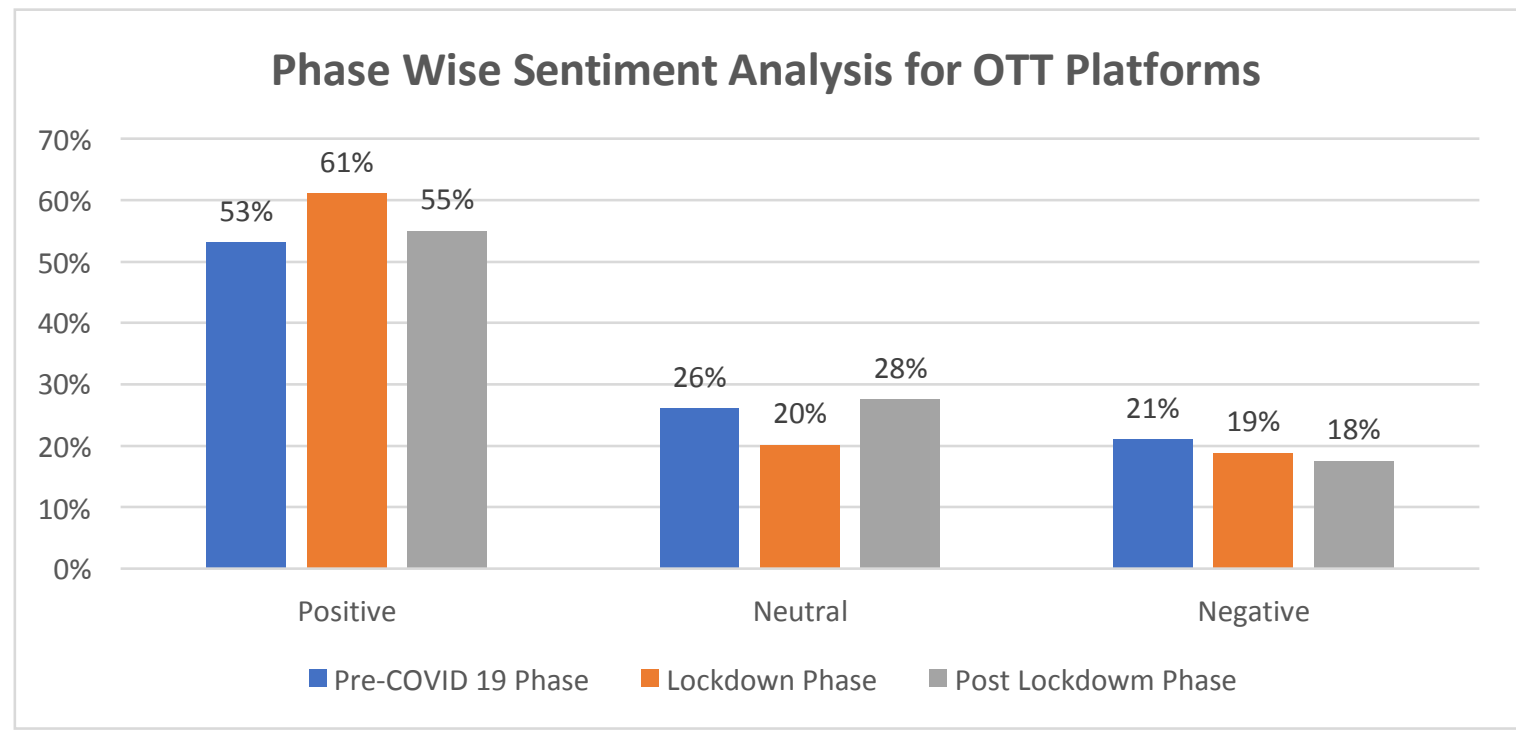

to the new way of life, digital consumption and

\section{Figure 3: Phase Wise Sentiment Analysis for OTT Platforms}

From the above bar graph, it is evident that during this COVID-19 pandemic people's engagement and people's opinion about the OTT platform has improved. According to the sentiment analysis result, during the lockdown period, positive engagement has increased $8 \%$ over the preCOVID-19 phase which has declined again post lockdown. Still, It can be concluded that over the period of time and due to this ongoing pandemic more people are positively adopting different OTT mediums, as the pre-COVID-19 positive score was $53 \%$ and in recent times (post Lockdown) it is $55 \%$.

As mentioned, that more people are reacting positively about OTT platforms as an entertainment medium, emotion classification can help us to gaze people's reactions more precisely. In recent times overall people's sentiment can be understood better through this technique. Emotion classification is a very insightful tool of sentiment analysis helps us to understand consumer sentiment patterns attached to a particular topic. In this paper, consumer sentiment is classified into 10 different sentiment paraments like positive, negative, trust, surprise, sadness, joy, fear, disgust, anticipation, and anger. 


\section{Emotion Classification for OTT Platform}

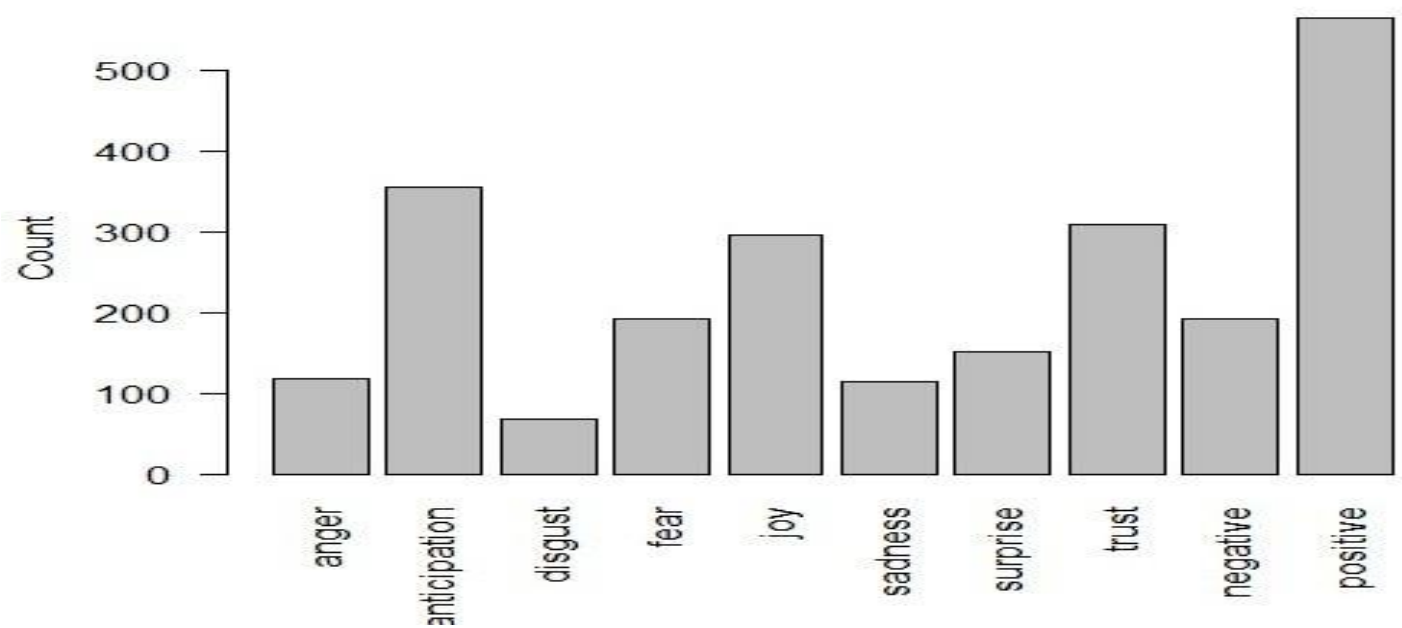

Figure 4: Emotion Classification of Twitter Dataset

The above emotion classification of the OTT platform further clarifies that people are willing to adopt the OTT platform because the count of positive words is more than 550 whereas the count of negative words is much lesser than positive words. It also showcases that people are ready to trust the OTT platform because the count of trustworthy words is more than 300 . Some fear is still attached to it, but consumers are more anticipated and have the joy to engage with the latest trend of entertainment media.

Now for understanding what stuck consumer's minds, most when they think or asked about OTT platforms, the word cloud is generated through data mining. Using the term definition matrix most common and frequently used buzz word about the OTT platform was identified. TermDocumentMatrix is a matrix that helps find the frequencies of a particular term used. It is very important since it helps us to understand the most frequent word used by consumers. Better visual insight was inferred through the word cloud of the OTT platform shown below. Word Cloud represents a cluster of the most frequent words used along with the topic "OTT platform". The size of any word in the word cloud depends on the frequency of that particular word. So higher the frequency larger the size of the word and the lower the frequency smaller the size of the word.

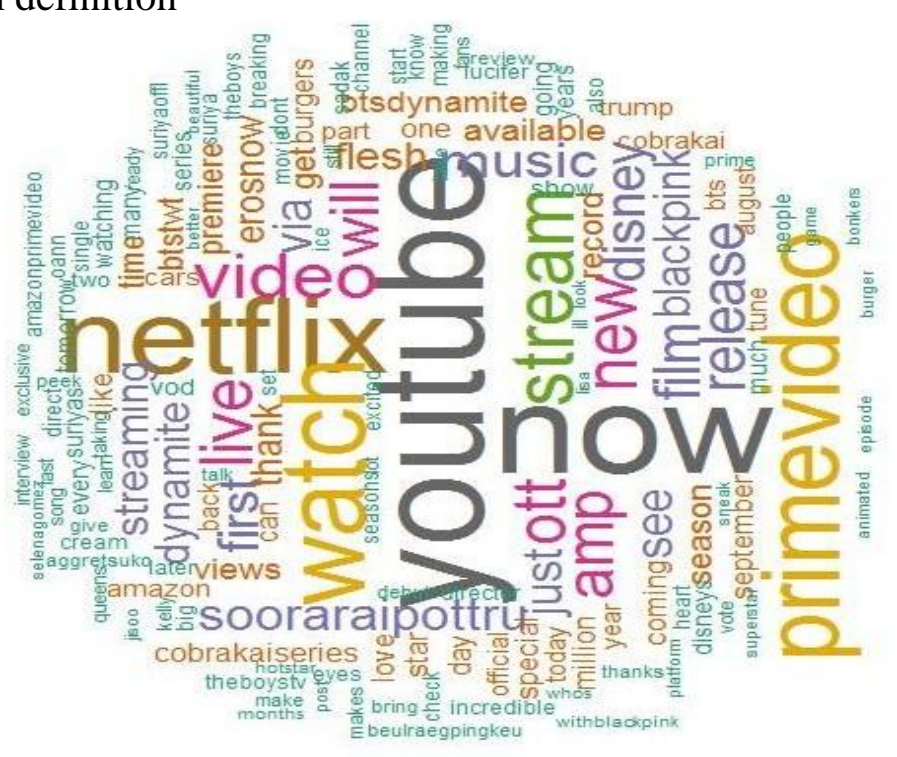

Figure 5: Word Cloud of Dataset (OTT tweets)

It can be easily understood from the above word cloud of the 'OTT platform' that 'YouTube', 
'Netflix', 'Amazon Prime Video', 'stream', 'new' 'release' are some of the most frequently used terms while discussing OTT platform. So, this word cloud indicates that among many OTT players YouTube, Netflix, and Amazon Prime video are the most popular among the consumer, and overall people are inclined towards online streaming and live shows offered by OTT players. Now a comparative consumer engagement analysis with these top 3 OTT players is done.

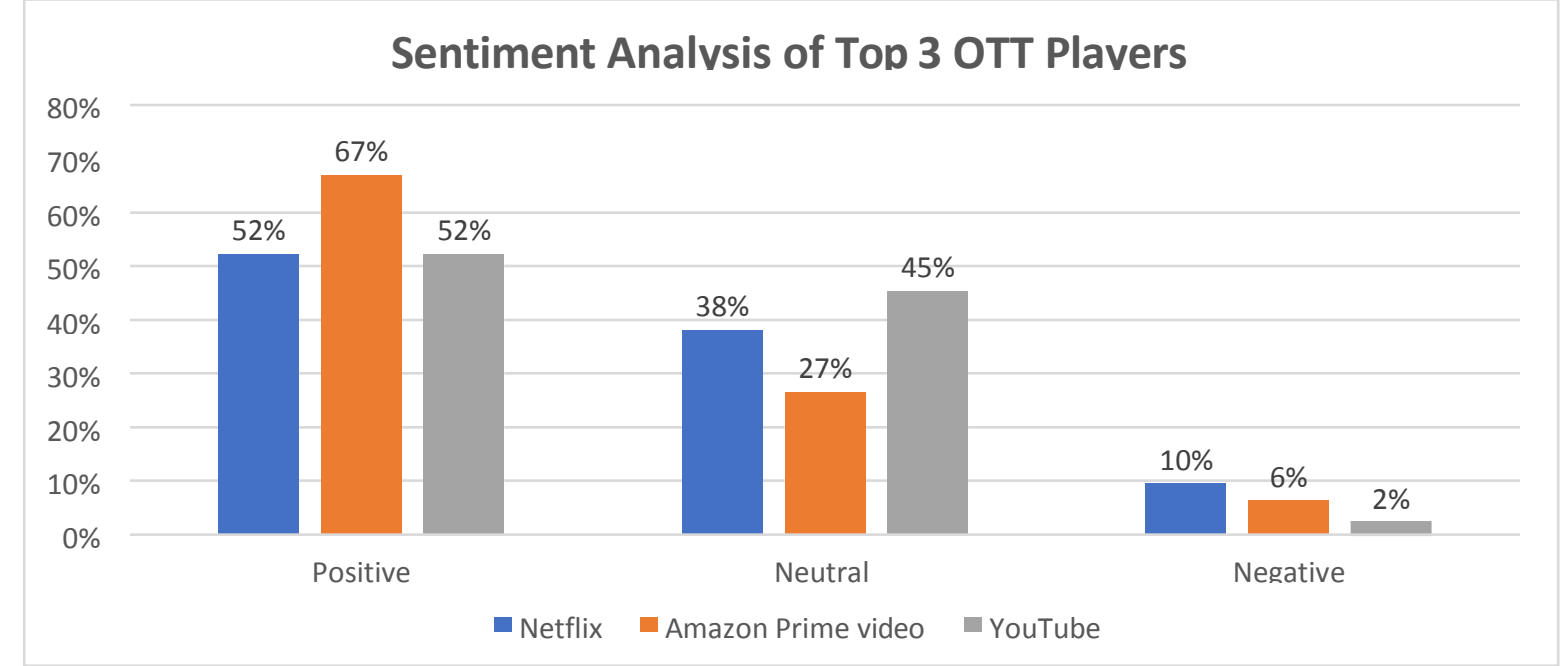

Figure 6: Sentiment Analysis of Top 3 OTT Players

According to the sentiment analysis result, people have more positive opinions and experience about Amazon Prime Video, and the other two platforms Netflix and youTube have got similar positive feedback. People have the least negative reaction and more generalized reaction towards YouTube while Netflix is the most criticized platform by the consumers.

\section{Limitations}

Technology and innovation are continuously shaping business models and accordingly consumer demand is also changing. Today a person can be satisfied with the current offering of a particular product or service, but it may happen that with changing time and market trend that person becomes dissatisfied with that product or service.

\section{Managerial Implications}

This study will be helpful to understand the consumer behavior towards the adaption of the OTT platform and customer inclination towards the current trend of entertainment. This research proves that consumers have a positive attitude about the OTT platform, and they are happy to adopt this on-demand entertainment model. This paper will surely motivate other researchers of the OTT platform or stakeholders who are working in this latest entertainment medium and definitely conclusion drawn from the research will be utilized for better customer satisfaction.

\section{Conclusion}

The current COVID-19 pandemic and different social distancing measured attached to COVID19 has not only changed economic activity and industries but has also led to a change in behavioral patterns in consumers, including that of digital consumption. As the media and entertainment industry is heavily dependent on many on-ground events, huge losses and slowdown is observed in this sector due to COVID-19. But to the contrary OTT media has boomed as a sustainable alternative of traditional Entertainment and Media industry. The ongoing COVID-19 pandemic has added a whole new momentum to the digital revolution of the media industry. The subscription revenue, adaption rate of OTT platform is rising because of the quality content, flexibility, and variety. Another significant reason behind the increasing popularity of OTT platforms is the multiscreen availability of content and the high rate of smartphones/smart-devices penetration around the globe. Along with lucrative features, different innovative business strategies taken by different OTT players (i.e. free subscription, extended period offers, multiple viewership facility) could result in a new normal and indulge some permanent shift in the media consumption habit of 
consumers. More people are inclining toward the OTT platform during this lockdown period and the overall consumer mindset towards the OTT platform is showcasing significant positive responses.

\section{References}

[1] Baldry, S. ;, Steingröver, M. ;, \& Hessler, M. A. (2014). The rise of OTT players: What is the appropriate regulatory response? http://hdl.handle.net/10419/101398www.eco nstor.eu

[2] Budzinski, O., \& Lindstädt-Dreusicke, N. (2018). The new media economics of videoon- demand markets: Lessons for competition policy. Ilmenau Economics Discussion Papers, 24(116), 1-51. http://hdl.handle.net/10419/184728

[3] Cambria, E., Schuller, B., Systems, Y. X.-I. I., \& 2013, U. (2013). New avenues in opinion mining and sentiment analysis. Ieeexplore.Ieee.Org, 28(2), 15-21. https://ieeexplore.ieee.org/abstract/documen t/6468032/

[4] Edosomwan, S., Prakasan, S., ... D. K.-J. of A., \& 2011, U. (2011). The history of social media and its impact on business. Minot.Com, 16(3), 79-91. http://www.minot.com/tom/SocialMedia.pdf

[5] Gaoshinde, M. (2020). Impact Of Embedded Marketing On OTT Platforms.

[6] Herrero-Subías, M. (2003). 4 Programmming and Direct Viewer Payment for Television. The Case of Canal Plus Spain J u n e 2003.

[7] https://dadun.unav.edu/bitstream/10171/366 26/1/201407 - 04 (2003) Programming and Direct Viewer.pdf

[8] Khatri, B. (2019). 4 Out Of 5 Smartphone Users Use At Least One OTT Entertainment. https://inc42.com/buzz/4out-of-5-smartphone-users-use-at-least-oneott-entertainment/

[9] Kohli, D. (2019). Looking into the future, over the top: OTT trends that will lead the way in 2019. https://yourstory.com/2019/02/looking-intofuture-top-ott-trends-2019

[10] Kokaram, A., Crinon, R., \& Catania, N. (2015). OTT (Over-The-Top) in 2015. 124(6), 2015- 2018.

[11] Lacy, L. (2017). Digital marketing spend to hit $\$ 118$ bn by 2021 , but budgets will shift to experiences | The Drum. https://www.thedrum.com/news/2017/01/26 /digital-marketing- spend-hit-118bn-2021budgets-will-shift-experiences-0

[12] Li, N., \& Wu, D. D. (2010). Using text mining and sentiment analysis for online forums hotspot detection and forecast. Decision Support Systems, 48(2), 354-368. https://doi.org/10.1016/j.dss.2009.09.003

[13] Madhani, R., \& Nakhate, V. (2020). Comparative Study Of Viewers 'Behaviour Over Traditional Television Channels And Over Ott Video Platforms In Maharashtra. 29(12), 1046-1057.

[14] Noam, E. M. (2016). Who Owns the World's Media?: Media Concentration and Ownership Around the World - Google Books. https://books.google.co.in/books?hl=en\&lr= $\& \mathrm{id}=\mathrm{kDfuCgAAQBAJ} \&$ oi=fnd $\& p g=P P 1 \&$ d $\mathrm{q}=$ Who + Owns + the + World $\% 27 \mathrm{~s}+$ Media $\% 3$ $\mathrm{F}+$ by + Noam $+2016 \&$ ots $=7$ NEeef3QG4\&sig $=$

JG23LWjd2jd_5Bm9vItNiG5UCKw\&redir _esc $=y \# v=$ onepage \&q $=$ Who Owns the World's Media\%3F by Noam 2016\&f=false

[15] Rashid, A. (2019). Hotstar Breaks Record With 25.3 Million Concurrent Viewers During India vs New Zealand World Cup Semi-Final | Entertainment News. https://gadgets.ndtv.com/entertainment/news /hotstar-world-record-25-3-million-viewersicc-cricket-world-cup-2019-india-newzealand-semi-final-ma-2069996

[16] Richwine, L., \& Rana, A. (2020). Netflix doubles expected signups but warns coronavirus boost may fade | Reuters. https://in.reuters.com/article/netflixresults/netflix-doubles- expected-signups- 
but-warns-coronavirus-boost-may-fadeidINKCN22407H

[17] Singh, D. (2020). Coronavirus Impact on OTT: How is coronavirus impacting the streaming platforms with an increasing appetite of viewers | The Financial Express. https://www.financialexpress.com/brandwag on/how-is-coronavirus-impacting-thestreaming-platforms-with-an-increasingappetite-of-viewers/1919916/

[18] Singh, P. (2019). New Media as a Change Agent of Indian Television and Cinema: A study of over the top Platforms. Journal of Content, Community and Communication, 9(2019), 131-137. https://doi.org/10.31620/JCCC.06.19/18

[19] Urgelles, A. (2017). The Threat of OTT for the Pay-TV Market. Current and Emerging Issues in the Audiovisual Industry, 19-38. https://doi.org/10.1002/9781119384632.ch2 